\section{Lines of Large Frequency Shift in the Raman Spectra of Crystals.}

Prof. F. Rasetti ${ }^{1}$ has deseribed lines in the Raman spectrum of fluorite and calcite, which show a remarkably large shift of about $7000 \mathrm{~cm}^{-1}$. Owing to the theoretical importance of this fact, which would indicate the co-operation of electronic transitions of considerable energy in the Raman spectra, I wish to point out that these lines can be explained as lines of the phosphorescence of gadolinium. It is a wellknown fact that natural fluorite nearly always contains rare earths. These earths, when excited by ultra-violet light or cathode rays, show a phosphorescence having a spectrum of very sharp lines. The spectrum of fluorite was examined by $G$. Urbain. ${ }^{2}$ The following table shows that the observed lines coincide with the lines of phosphorescence of gadolinium.

\begin{tabular}{|c|c|c|}
\hline $\begin{array}{c}\Delta \nu \text { from } \\
\mathrm{Hg} \lambda 2537 .\end{array}$ & $\begin{array}{c}\text { Observed } \\
\text { lines. } \\
\lambda\end{array}$ & $\begin{array}{c}\text { Lines of gado- } \\
\text { linium, after } \\
\text { Urbain. } \\
\lambda\end{array}$ \\
\hline 7256 & 3109 & 3108 \\
7273 & 3110 & 3110 \\
7285 & 3112 & 3113 \\
7298 & 3113 & 3115 \\
\hline
\end{tabular}

The agreement is as good as the limit of error in the exact measurement of the lines of phosphorescence will allow. In addition, Urbain observed other lines between $3118 \mathrm{~A}$. and $3147 \mathrm{~A}$. That these lines were not observed in the Raman spectrum is easily understood. These lines coincide partly with the strong lines of mercury in this region, and further, Urbain showed that the lines between $3147 \mathrm{~A}$. and $3132 \mathrm{~A}$. disappear when the amount of gadolinium in the fluorite becomes very small. Conversely we can conclude that the material examined by Prof. Rasetti contained only a very small quantity of gadolinium.

An analysis of the spectrum of calcite is not possible, since the spectrum of phosphorescence of gadolinium in calcite has not yet been examined. The known spectrum in calcium oxide shows also in the spectral region under consideration the main lines of gadolinium.

Hence in the examination of materials which are likely to show a phosphorescence spectrum of sharp lines, the appearance of such lines, as well as the lines of the Raman spectrum, is, if they are excited with ultra-violet light, always possible.

It can also be seen from the above, that similar lines of large frequency shift are not recognisable in the examination of other materials, since they most probably contain no rare earths.

\section{Physical Laboratory, \\ University of Marburg, July 24 .}

1 Natere, A pril 25, 1931, p. 626.

2 Ann. de chim., 1909, and "Handb. d. Experimentalphysik", 23, $484 ; 1928$.

\section{The Fine Structure of Spectral Lines of Light Scattered by Liquids.}

Gross ${ }^{1}$ has investigated by means of an echelon grating the fine structure of spectral lines scattered by liquids, and has found that in addition to the lines scattered without change of wave-length there appear new lines symmetrically situated relatively to the primary ones. The number of those new lines depends from the substance investigated, and the distance between them depends upon the angle formed by the incident and the dispersed rays.
Similar investigations were carried out by Vacher. ${ }^{2}$ He used for that purpose a Fabry Perot interferometer. His observations, however, do not confirm Gross's results. Though he found that the primary line is widened by increasing the angle between the incident beam and the direction of observation, yet he states that this widening takes place only towards the long wave-length. Also, he did not observe the appearance of single lines.

The discordance between the results mentioned above induced me to undertake a similar investigation, using a different experimental arrangement. I put the vessel with the liquid investigated inside the mercury lamp, which had the form of a ring (the detailed description of the apparatus will be given in a paper which will be published in Bull. de l'Acad. Pol.). A current of $4 \frac{1}{2} \mathrm{amp}$. passed through the lamp burning at $110 \mathrm{v}$. The scattered light after passing through a monochromator fell on a Lummer-(Yehrcke plate. I used in my experiments two different quartz plates of thicknesses of $3.92 \mathrm{~mm}$. and $6.888 \mathrm{~mm}$. and lengths of $13 \mathrm{~cm}$. and $20 \mathrm{~cm}$. respectively. In the case of benzene there does not appear in the scattered light any new line, though the components observed by Gross were of the same intensity as the primary component. Taking in consideration Gross's data, those lines should appear half-way between the neighbouring orders of the primary line. The spectra of the scattered light in both cases, benzene and white paper, differ only by the continuous background and by a slight widening of the lines which appears in the case of benzene. I obtained similar results with water.

The same experiments were undertaken with a different arrangement of apparatus; I irradiated the liquid at a definite angle $\left(90^{\circ}\right)$. In this case also the spectrum does not alter in any noteworthy way.

Since Vacher and I are not able to confirm the phenomenon observed by Gross by means of other interferometric apparatus (Fabry Perot interferometer and Lummer-Gehrcke plate), hence a more accurate investigation of that problem, and the explanation of the difficulties involved, would be desirable. St. Rafalowski.

Physical Laboratory of the Society of Sciences and Letters, Warsaw, July 8.

1 Gross, Nature, 126, 201, 400, $603 ; 1930$.

2 Vacher, Phys. Ber., 12, 1044 ; 1931. C. R., 191, 1121-1123; 1930.

\section{Vitamin A and the Antimony Chloride Reaction.}

For the determination of vitamin A by means of the reaction of Carr and Price, the blue colour of the mixture is estimated with the Lovibond tintometer. If analysed by the spectroscope, the blue mixture shows two absorption-bands at $572 \mu \mu$ and $610 \mu \mu$ respectively (Morton). The blue colour depends only on the intensity of the 610 band.

Recently Morton and Heilbron have announced that the strength of different vitamin A preparations corresponds better with the extinction of the mixture in the $572 \mu \mu$ region than in the $610 \mu \mu$ region.

We can confirm this by the following facts: By treating a vitamin A preparation from saponified cod liver oil or saponified extract of cow's liver with some drops of furan, methylfuran, pyrrol, indol, or skatol (all substances of related chemical structure) and then adding antimony chloride, the mixture turns purple instead of blue, and in the spectroscope the $610 \mu \mu$ band is no longer seen, while the $572 \mu \mu$ band remains unaltered. The physiological activity is unimpaired and the preparation (without antimony

No. 3229, VoL. 128] 